\title{
A Classical Approach to Nuclear Instability
}

\author{
David L. Selke
}

\section{ABSTRACT}

We extend a classical model of nuclei to include geometry as well as electronlike shells, then apply the model to the moments leading up to nuclear decay. The model leads to the well-known half-life or constant probability of a given nucleus to decay in a given time.

Keywords: classical, electromagnetics, instability, nuclear.

\author{
Published Online: October 12, 2021 \\ ISSN: $2684-4451$ \\ DOI : 10.24018/ejphysics.2021.3.5.109
}

D. L. Selke*

New Model Arts, Chandler, AZ, USA.

(e-mail: dselke@hotmail.com)

*Corresponding Author

\section{INTRODUCTION}

Classical physics was abandoned by mainstream physicists for radically new principles in the beginning of the 20th century. But what if, with more theoretical effort, nature could be explained classically after all? We follow Randell Mills' Grand Unified Theory of Classical Physics (GUTCP) in the tradition of giving classical models a much deserved second look. His results [1] in physics-based chemistry are unmatched by mainstream theories and indicate that he is on the right track. Here we will add more detail to a developing nuclear model based on Mills' nucleon charge distributions.

\section{SHELl MODEL}

It is a strength of GUTCP-based nuclear models that they are strongly constrained by known physics, that is, by Maxwell's Equations and Newton's Laws. We previously argued [2], based on electrostatics and binding energy, for a nuclear model consisting of shells which are full when an alpha particle (two protons and two neutrons) occupies them. Starting from the GUTCP nucleon, an obvious way to combine them is to make the center and radius of each nucleon the same. Such a single-radius nucleus can be seen as a limiting case of a nucleus with concentric shells of different sizes. In each case, the net positive charge of each shell repels the net positive charge of each other shell. However, the vector directions of these pointwise repulsions are oriented in every direction, so that they tend to cancel out. With a single radius for all shells, however, a perturbation to one shell moves its center away from the common center, and moves its surface, on one side of the nucleus, outside the common radius, and on the other side of the nucleus, inside the common radius. This leads to a net repulsion on the perturbed shell in the direction of the perturbation. As that shell's center and the common center move apart, more of the perturbed shell is exposed outside the common radius, and the repulsion becomes stronger. In this way, any small perturbation of a nuclear model having a common radius and center for all nucleons leads to the decay of the nucleus.

On the other hand, the more general model which has concentric shells with different radii does not necessarily decay in response to any perturbation. Consider a concentric model for Carbon-12, with inner, middle, and outer Helium4 shells. Perturb the middle shell "up" a small amount. This brings the middle shell closer to the outer shell at the top, brings the middle shell further from the inner shell at the top, brings the middle shell closer to the inner shell at the bottom, and brings the middle shell farther from the outer shell at the bottom. All four of these changes either increase the repulsion in the "down" direction or decrease the repulsion in the "up" direction, that is, all four of these effects oppose the perturbation and tend to restore the original position. A similar result holds for perturbations of the inner and outer shells. Recall that in GUTCP the nucleon charges are not uniform, so it is not the case that all the forces on a given shell cancel for a concentric nuclear shell model.

\section{DECAY CONDITION}

GUTCP states that nucleons rotate at the speed of light [1]. We have interpreted this to mean that they rotate as a globe with the speed of light at the equator [3]. It follows that inner shells, with the same linear velocity at the equator, have greater angular velocity and thus shorter periods of rotation. The charge distribution of both proton and neutron is such that there is a lobe of maximum positive charge between the axis and the equator and opposite that, a lobe of maximum negative (or minimum positive) charge. Even when combined into a complete shell (alpha particle), there are extrema of charge that rotate as the nucleons rotate. Because of these extrema rotating at differing periods for different radii, the shells perturb one another through the electric force even without an external perturbation. At a gross level, the period at which a system containing two shells returns to the initial state of the phase relationships of its charge extrema is equal to the least common multiple of the periods of each shell (that is, a multiple of both periods, ensuring that each of them has 
returned to its original phase at the same time as the other). More shells add additional terms, making the period of the whole system longer.

Most nuclides are unstable. Using the experimental constraint that half-lives are constant, that is, the chance for a nucleus to decay in one second is constant with time, we will develop the model further. Since the underlying GUTCP theory is deterministic, it will be possible to "wind back the clock" from the moment decay products are formed to a time some fraction of a second earlier when only the shells but not the decay products existed. The changeable properties of these shells, which are assumed to determine when they decay or do not decay, are the phase of rotation of each nucleon and the center of each nucleon. The charge distributions and radii might also change, but for the present purpose such changes are neglected. The Be- 8 decay into two alpha particles might be treated directly in an electromagnetic simulation, starting at a time when the nucleus holds together for the moment, through the time when the two daughter nuclei are separated. However, many light nuclei decay by a form of beta decay in which an electron or positron must be created, a proton or neutron is changed into the other, and an (anti-)neutrino is emitted. Though it would be desirable to know the intermediate states of this creation of particles and its physical principles, for now it must remain mysterious. Instead of explaining beta decay itself, we will focus on a state just prior to decay, which leads immediately to the decay, which we call the "decay condition". We will consider separately a "gross behavior" in which the phases and centers of the nucleus periodically return to any earlier set of values, and a "fine behavior" which modifies this trend by small changes stemming from effects we neglected or from external influences.

\section{NEAR APPROACH}

An estimate for the $\mathrm{He}-4$ radius is $1.67824 \mathrm{e}-15 \mathrm{~m}$. [4]. We will use this as a rough estimate for a generic unstable nucleus, for concreteness. Using this radius and the speed of light we compute the period at which the decay condition recurs. The period is very small, at $3.51 \mathrm{e}-23$ seconds, and the corresponding frequency is very large, at $2.85 \mathrm{e}+22 \mathrm{~Hz}$. Instead of saying the phase relationships exactly recur $2.85 e+22$ times a second, we invoke the fine behavior and say that the phase relationships nearly recur at this rate. That is, there is a near approach to the decay condition $2.85 \mathrm{e}+22$ times a second. Based on the details of each approach, the nucleus may decay or may not decay. The overall chance of decay would incorporate the number of near approaches in a time period as well as "how close" they were to the decay condition. Instead of a fixed particle decay time, we can relax the assumption that the decay condition immediately precedes a decay and see it as just another "near approach" or "decay opportunity" that an unstable nucleus routinely generates. An example: let one shell have a period of 3 and another a period of pi. Then any phase relationship between them (including a decay condition) will recur at period $3 *$ pi. Now let a third shell perturb the first two shells. Now the phases at $3 *$ pi are only close to the phases at 0 , no longer exact. In a second, many near approaches occur, some more and some less likely to lead to decay. But the chance per second of decay becomes constant by the law of large numbers. Thus, an overview of the physics of the concentric shell nuclear model based on GUTCP agrees with the experimental result that half-lives are constant for a given decay.

\section{CONCLUSION}

We proposed a classical concentric shells nuclear model which led to multiple interacting periods of the shells. This model accommodates the experimental result that the chance of decay of one nucleus in one second is constant for that type of nucleus. Equivalently, we can say that the model supports the half-life behavior of unstable nuclei.

\section{REFERENCES}

[1] R. Mills, The Grand Unified Theory of Classical Physics, East Windsor, NJ.: Brilliant Light Power. January 2020. https://brilliantlightpower.com/book-download-and-streaming/.

[2] D. Selke, "Toward Classical Models of Nucleons and Nuclei," European Journal of Applied Physics, vol. 2, pp. 1-2, June 2020. https://ej-physics.org/index.php/ejphysics/article/view/10.

[3] D. Selke, "Numerical Computation of Nucleon Properties in Mills' Grand Unified Theory of Classical Physics," Physics Essays, vol. 3, pp. 358-373, August 2015. https://physicsessays.org/browse-journal2/product/1378-14-david-1-selke-numerical-computation-of-nucleonproperties-in-mills-grand-unified-theory-of-classical-physics.html.

[4] J. Krauth et al., "Measuring the Alpha-Particle Charge Radius with Muonic Helium-4 Ions," Nature, pp. 527-531, January 2021. https://www.nature.com/articles/s41586-021-03183-1. 